\title{
Étude de la qualité des eaux usées des deux retenues du bassin du Gourou au carrefour de l'Indénié à Abidjan en 2012.
}

Coulibaly-Kalpy Julien ${ }^{*}$, Koffi Kouadio Stéphane ${ }^{2}$, Yéo Alain ${ }^{1}$, Yah Kouadio Léonard ${ }^{3}$, Bamba Aboubacar', Coulibaly Emmanuel2, Kacou-N'douba Adèle ${ }^{1,2}$, Dosso Mireille ${ }^{1,2}$.

1 Institut Pasteur de Côte d'Ivoire, 01 BP 490 Abidjan

2 Université Félix Houphouët-Boigny, BP V 34 Abidjan

3 Projet RETROCI, CHU Treichville, 01 BP V 03 Abidjan 01

Auteur Correspondant : Coulibaly-Kalpy Julien*; Institut Pasteur de Côte d'Ivoire, 01 BP 490 Abidjan ; email :

jc_kalpy@yahoo.fr

Original submitted in on $1^{\text {st }}$ November 2016. Published online at www.m.elewa.org on $31^{\text {st }}$ May 2017 https://dx.doi.org/10.4314/jab.v113i1.3

\section{RÉSUMÉ}

Objectif : Le défaut de filière de traitement des eaux usées a conduit à la réalisation de ce travail avec pour objectif d'Étudier la qualité bactériologique des eaux usées dans les retenues de l'Indénié contiguës aux grands collecteurs du bassin du gourou.

Méthodologie et Résultats : Les échantillons ont été prélevés dans des bouteilles en verres et analysées suivant les méthodes de microbiologies classiques. Dans la retenue A, la concentration moyenne de coliformes totaux était de 2,74.107 UFC/100mL. Celles des coliformes thermotolérants, $E$. coli et les streptocoques fécaux, ont été respectivement de 1,49.107 UFC/100mL, 1,22.107 UFC/100mL et de 9,19.106 UFC/100mL. Dans la retenue B, la concentration moyenne de Coliformes totaux était de 2,98.107 UFC/100mL. Considérant les Coliformes thermotolérants, $E$. coli et les streptocoques fécaux, les charges moyennes ont été respectivement de 2,19.107 UFC/100mL, 1,72.107 UFC/100mL et de 1,32.107 UFC/100mL.

Conclusion et application des résultats : Les eaux des deux retenues étudiées présentent une mauvaise qualité bactériologique eue égard aux concentrations des germes indicateurs de pollution fécale. En effet les concentrations en $E$. coli, Streptocoques fécaux et coliformes totaux étaient largement supérieurs aux normes OMS pour les eaux usées destinées à être rejetée dans l'environnement sans risque sanitaire. II apparait alors plus que nécessaire de créer des filières de traitement de ces eaux usées avant leur rejet dans le milieu naturel. Ce qui pourrait réduire les risques de pollution environnementale d'une part et sanitaire d'autre part. Les résultats de ce travail posent aussi le problème du mode d'utilisation des collecteurs et des retenus d'eaux usées qui passent pour être de vrais dépotoirs.

Mots clefs : Eaux Usées, pollution, risque sanitaire, indicateurs 
Study of the quality of wastewater in two reservoirs of the Guru basin at the crossroads of Indénié in Abidjan in 2012

\begin{abstract}
Objective: Failure of wastewater treatment process has led to the study of the bacteriological quality of wastewater in reservoirs of Indénié adjacent to major collectors of Guru basin

Methods and Results: The water samples were collected in glass bottles and analyzed according to the methods of conventional microbiology. In the reservoir A, the average concentration of total coliform was $2.74 .10^{7} \mathrm{cfu} /$ $100 \mathrm{~mL}$. Those of thermotolerant coliform, E. coli and faecal streptococci, were respectively $1.49 .10^{7} \mathrm{cfu} / 100 \mathrm{~mL}$, $1,22.10^{7} \mathrm{cfu} / 100 \mathrm{~mL}$ and $9,19.10^{6} \mathrm{cfu} / 100 \mathrm{~mL}$. In the reservoir B, the average concentration of total coliforms was $2.98 .10^{7} \mathrm{cfu} / 100 \mathrm{~mL}$. Considering the thermotolérants coliforms, E. coli and faecal streptococci, the average charges were respectively $2.19 .10^{7} \mathrm{cfu} / 100 \mathrm{~mL}, 1.72 .10^{7} \mathrm{cfu} / 100 \mathrm{~mL}$ and $1.32 .10^{7} \mathrm{cfu} / 100 \mathrm{~mL}$.

Conclusion and application of results: The waters studied, exhibit poor bacteriological quality according to the concentrations of bacteria indicating fecal pollutions. Indeed concentrations of E. coli, fecal streptococci and total coliforms were significantly higher than WHO standards for wastewater intended to be released to the environment without risk to health. So, the need of wasterwater treatment prior to discharge into the natural environment.
\end{abstract}

Keywords: Sewage, pollution, health risks, indicator

\section{INTRODUCTION}

Les rejets des eaux usées dans l'environnement peuvent être source d'infections provenant d'agents polluants tels les microorganismes pathogènes d'origines diverses (Anonyme, 2012). Selon l'Organisation Mondiale de la Santé, chaque année près de 1,8 million de personnes dont $90 \%$ d'enfants de moins de cinq ans meurent de maladies diarrhéiques (Fontaine, 2008). Or, à l'échelle mondiale, l'immense majorité des maladies diarrhéiques sont imputables à la mauvaise qualité de l'eau de boisson et à un assainissement insuffisant des eaux usées (Servais et al, 2009). Si l'assainissement des eaux usées est devenu un impératif pour les Sociétés modernes, les pays africains connaissent, de graves difficultés d'une part pour l'alimentation en eau et d'autre part pour l'évacuation et le traitement des eaux usées (Wayou, 2010). II est à noter que $78 \%$ des villes africaines ne disposent d'aucun service formel d'évacuation et de traitement des eaux usées. Lorsqu'elles existent, elles sont dépassées pour la plupart (Akossi, 2011). Dans ces pays, l'insuffisance des réseaux d'assainissement et la non connexion de la majorité de la population (plus de 30\% à Abidjan) à l'égout font qu'on observe des rejets d'eaux usées sans traitement dans le milieu naturel. (Wayou, 2010). La Côte d'Ivoire dans sa politique de développement a consenti à la construction de deux retenues pour réguler le flux d'eaux usées en provenance des différents quartiers au nord de la ville d'Abidjan (Ouattara, 2005). Ces retenues sont nées du projet de gestion intégrée du bassin du Gourou (Cl-FAD, 2010). Malheureusement ces retenues mal entretenues n'arrivent pas à jouer leur rôle premier et connaissent des dysfonctionnements susceptibles d'entrainer des maladies. Bien que la majorité des bactéries présente dans les eaux usées ne soient pas pathogènes, certains pathogènes comme Salmonella, probablement amené par les activités anthropiques sont impliqué depuis longtemps dans les maladies d'origine hydrique principalement dans les pays en développement (Kotloff et al, 2012). Dans de nombreux pays, la surveillance de la qualité des eaux usées lorsqu'elle est réalisée, se limite à l'estimation du niveau de microorganismes indicateurs de pollution (Levantasi et al, 2010). Aussi a-t-il été décidé de réaliser cette étude bactériologique avec pour principal objectif d'étudier la qualité bactériologique des eaux usées dans les retenues de l'Indénié contiguës aux grands collecteurs du bassin du gourou. 


\section{MATÉRIELS ET MÉTHODE}

Cadre de l'étude : Les échantillons ont été récoltés dans les retenues contiguës aux collecteurs du bassin du gourou. Les analyses de cette étude se sont déroulées à I'Unité de Chimie et de Microbiologie Environnementale de l'Institut Pasteur de côte d'Ivoire.

Présentation de la zone de prélèvement : Le Bassin du Gourou couvre une superficie d'environ 28,6 km2. II s'étend du Nord au Sud sur environ $9 \mathrm{~km}$. D'une largeur moyenne de $3 \mathrm{~km}$, il est limité à l'Est par le prolongement du boulevard Latrille vers le quartier des II Plateaux et à l'Ouest par la ligne du chemin de fer (Adjamé-Anyama) (Cissé et al , 2011). La présente étude a porté sur deux retenues dont une jouxtant le Lycée Technique d'Abidjan
(Retenue A) et l'autre le siège du journal Fraternité Matin (Retenue B) :

- la retenue $\mathrm{A}\left(05^{\circ} 23^{\prime} 13,6^{\prime \prime} \mathrm{N}\right.$ et $\left.04^{\circ} 00^{\prime} 22,0^{\prime \prime} \mathrm{W}\right)$, reçoit les eaux résiduaires urbaines qui proviennent d'AboboSud, Williamsville, Camp de Gendarmerie d'Agban, des deux plateaux Cocody et Cocody-Lycée Technique.

- la retenue $B\left(05^{\circ} 22^{\prime} 42,7^{\prime \prime} \mathrm{N}\right.$ et $\left.04^{\circ} 00^{\prime} 20,6^{\prime \prime} \mathrm{W}\right)$ reçoit les eaux résiduaires urbaines des zones de la grande Mosquée d'Adjamé-Gare, Adjamé-Bracodi, Adjamé-220 logements. Plateau Indenié et du quartier de FraternitéMatin. Les eaux usées provenant du bassin du gourou transitent donc dans ces deux retenues avant de se jeter dans la lagune Ebrié. (Akossi, 2011)

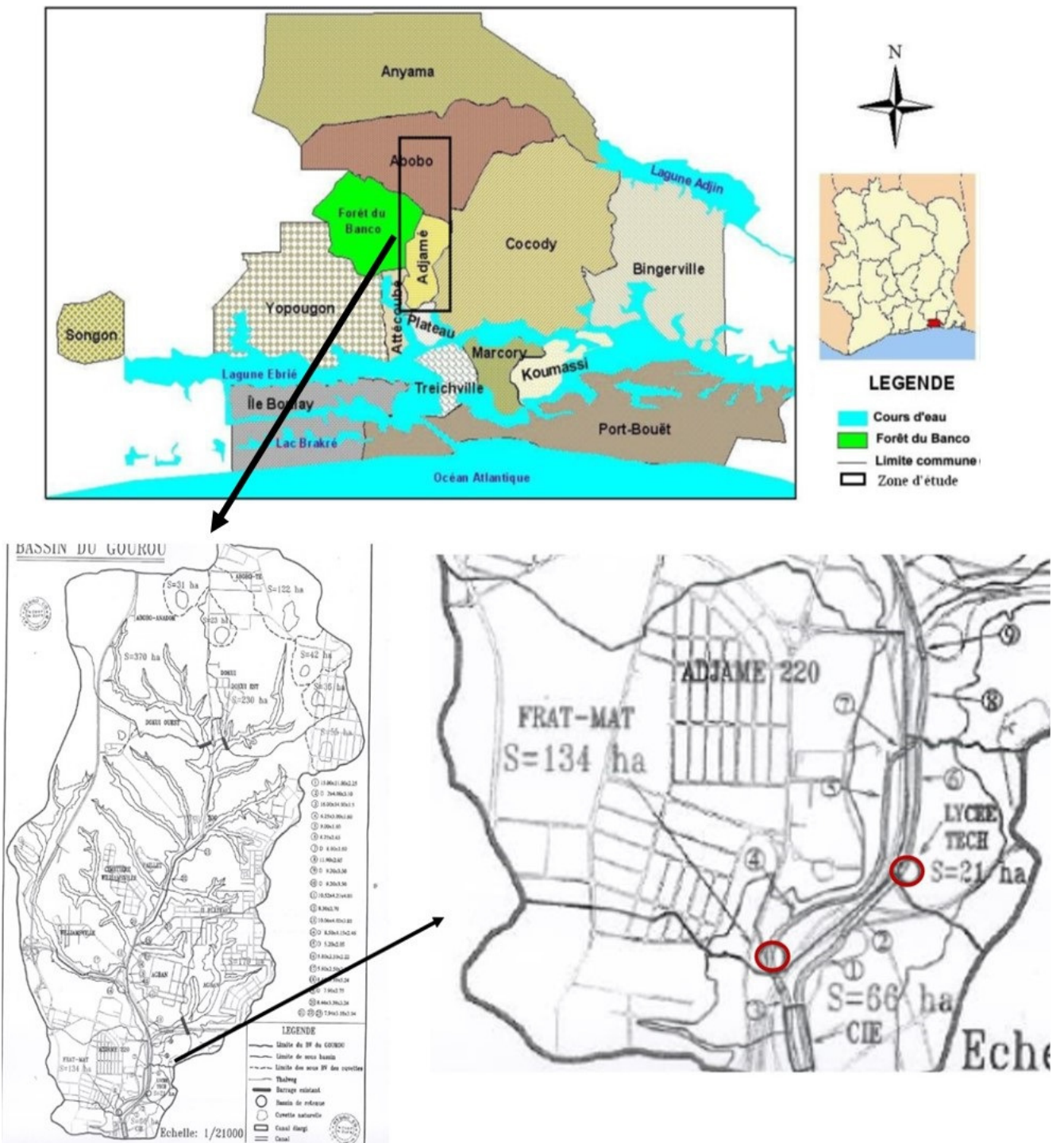

Figure 1: Carte du bassin du Gourou et des retenues étudiées modifiée. (Image : Kalpy J. COULIBALY) 
Échantillonnage : L'étude a porté sur les eaux usées contenues dans les deux retenues ci-dessus citées. Un plan d'échantillonnage précis n'a pas pu être établi car cette étude est la première phase d'une surveillance continue des retenues. Cependant des points ont été choisis en raison de l'accessibilité.

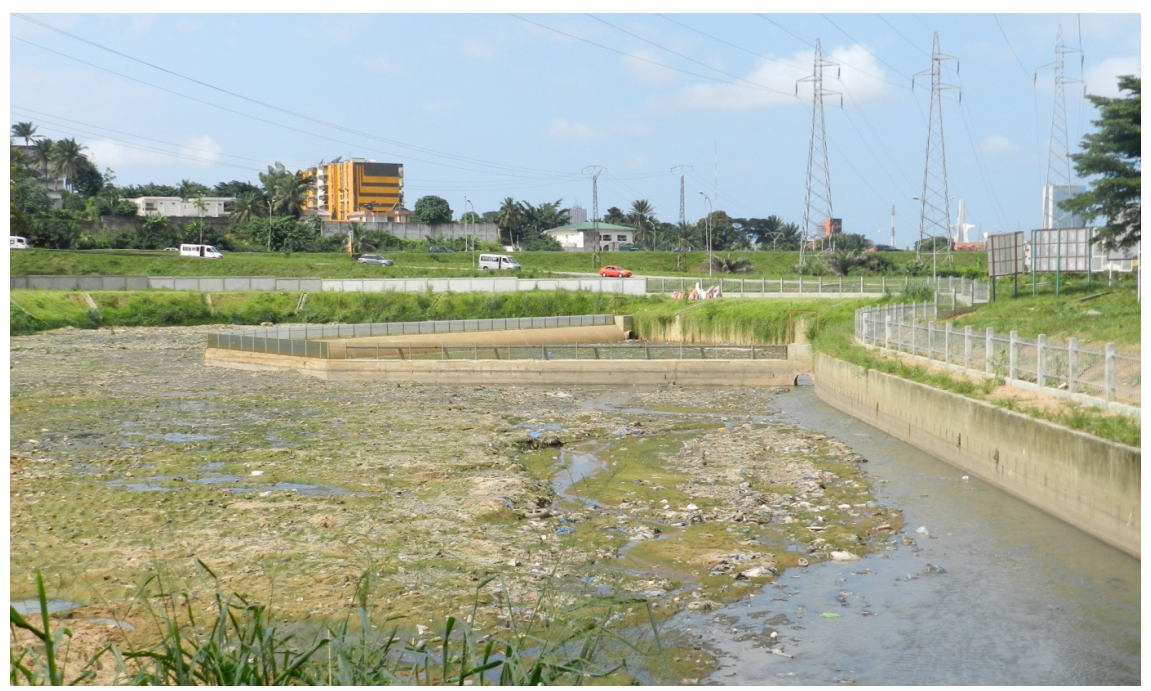

Figure 2: Vue générale retenue A Lycée Technique

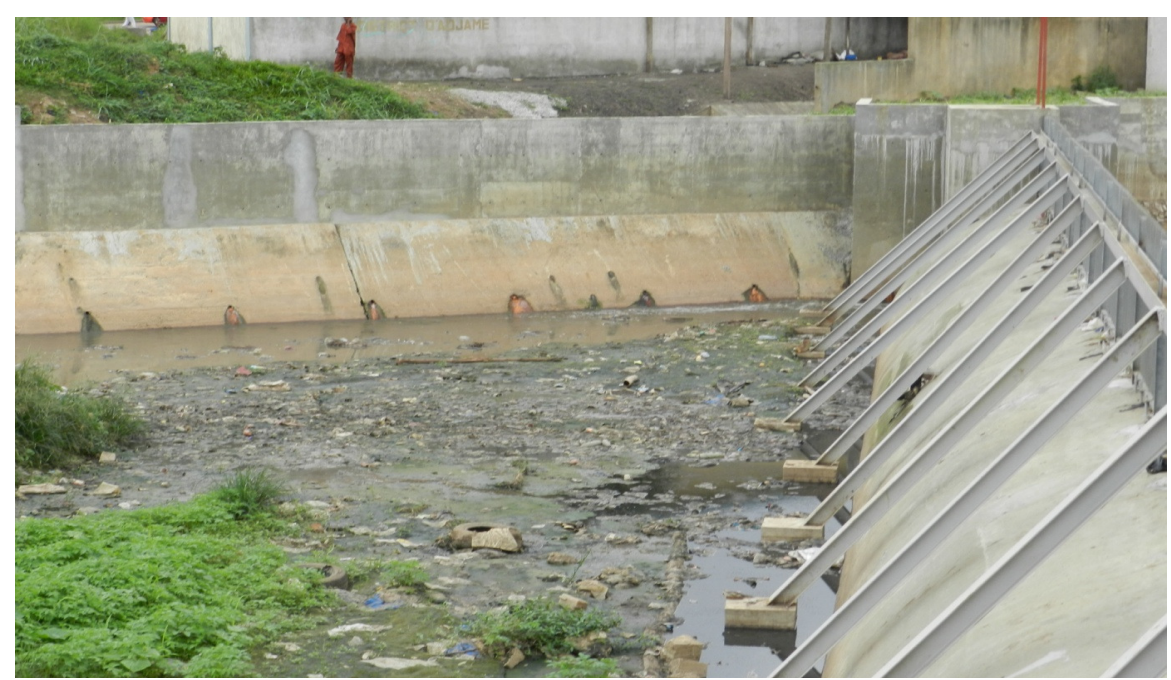

Figure 3: Vue générale Retenue $B$ Fraternité Matin

Méthode : L'échantillonnage s'est déroulé sur deux mois (2 Juin 2012 au 2 Aout 2012) période couvrant la grande saison des pluies, à raison de deux campagnes par semaines. Les prélèvements ont été effectués entre $8 \mathrm{~h}$ et 9 h30mn, suite à l'abondance des activités ménagères (Cissé et al , 2011). Les eaux usées ont été prélevées dans une bouteille en verre de $500 \mathrm{~mL}$ préalablement rincée avec l'eau à prélever. Les échantillons sont alors disposés dans une glacière contenant des accumulateurs de froid afin d'assurer le transport des échantillons à une température proche de $+4^{\circ} \mathrm{C}$.

Analyse au laboratoire
Identification des bactéries: Après avoir laissés les échantillons à décanter à la température du laboratoire pendant 20 minutes environ, un aliquot de $50 \mathrm{~mL}$ de chaque échantillon a été conservé à $-80^{\circ} \mathrm{C}$ au centre des ressources biologiques de l'IPCI. Une quantification des germes indicateurs de pollution fécale a été réalisée. Les coliformes totaux(CT), coliformes fécaux (CF) ou coliformes thermo tolérants ( $C T h$ ), streptocoques fécaux (SF) ont donc été recherchés et dénombrés. Les méthodes utilisées sont celles regroupées dans le tableau ci-dessous (Tableau 1) 
Tableau 1: Résumé des méthodes d'identification de microorganismes utilisées.

\begin{tabular}{|c|c|c|c|c|c|}
\hline Microorganismes & Milieu de culture & $\begin{array}{l}\text { Mode } \\
\text { d'ensemencement }\end{array}$ & $\begin{array}{l}\text { Durée } \\
\text { d'incubation }\end{array}$ & $\begin{array}{l}\text { Observation } \\
\text { particulière }\end{array}$ & Références \\
\hline E. coli & $\begin{array}{l}\text { Gélose Tryptone Bile } \\
\text { Xylène (TBX) pour } \\
\text { analyse de l'eau } \\
\text { Rapide E-Coli } 2 \text { (REC2) } \\
\text { pour analyse de la laitue }\end{array}$ & étalement & $\begin{array}{l}24 \mathrm{~h} \\
37^{\circ} \mathrm{C} \text { ou } 44^{\circ} \mathrm{C}\end{array}$ & $\begin{array}{l}\text { Colonies bleues ou } \\
\text { colonies violettes } \\
\text { Identification } \\
\text { biochimique à travers le } \\
\text { portoir }\end{array}$ & $\begin{array}{l}\text { uNorme ISO } \\
\text { s/NP } 16649 .\end{array}$ \\
\hline Coliformes totaux & $\begin{array}{l}\text { Gélose lactose biliée au } \\
\text { cristal violet et au rouge } \\
\text { neutre (VRBL) ou gélose } \\
\text { Mac Conkey }\end{array}$ & $\begin{array}{l}\text { ensemencement } \\
\text { edans la masse }\end{array}$ & $24 \mathrm{~h}$ à $37^{\circ} \mathrm{C}$ & Colonies rouges & $\begin{array}{l}\text { méthode NF } \\
\text { ISO } 4832 .\end{array}$ \\
\hline $\begin{array}{l}\text { Coliformes thermo } \\
\text { tolérants }\end{array}$ & $\begin{array}{l}\text { Gélose lactose biliée au } \\
\text {-cristal violet et au rouge } \\
\text { neutre (VRBL) ou gélose } \\
\text { Mac Conkey }\end{array}$ & $\begin{array}{l}\text { ensemencement } \\
\text { edans la masse }\end{array}$ & $24 \mathrm{~h}$ à $44^{\circ} \mathrm{C}$ & Colonies rouges & $\begin{array}{l}\text { norme NF V } \\
08-060\end{array}$ \\
\hline $\begin{array}{l}\text { Streptocoques } \\
\text { fécaux }\end{array}$ & $\begin{array}{l}\text { Gelose Bile Esculine } \\
\text { Azide (BEA) }\end{array}$ & étalement & $24 \mathrm{~h}$ à $37^{\circ} \mathrm{C}$ & Colonies noires & $\begin{array}{l}\text { norme ISO } \\
7899-1: 1984 \\
\end{array}$ \\
\hline
\end{tabular}

\section{RÉSULTATS}

II a été réalisé 112 prélèvements à raison de 56 par retenue pour un total de 14 sorties. Les coliformes totaux, E. coli et les streptocoques fécaux ont été mis en évidence dans tous les échantillons.

\section{Germes indicateurs de contamination fécale des eaux usées des retenues}

Évolution des germes indicateurs de pollution fécale de la retenue A: Dans la retenue A, la concentration moyenne de coliformes totaux était de $2,74.10^{7}$ UFC/100mL ( $\min =1,06 \cdot 10^{7} ; \max =7,05 \cdot 10^{7}$ UFC/100mL). Quant aux coliformes thermololérants, $E$. coli et les streptocoques fécaux, les charges moyennes ont été respectivement de $1,49.10^{7} \mathrm{UFC} / 100 \mathrm{~mL}\left(\mathrm{~min}=1,36.10^{6}\right.$; $\max =5,17.10^{7} \quad$ UFC/100mL), $1,22.10^{7} \quad$ UFC $/ 100 \mathrm{~mL}$ ( $\min =6,7.10^{5} ; \max =4,85.10^{7} \mathrm{UFC} / 100 \mathrm{~mL}$ ), et de $9,19.10^{6}$ UFC/100mL ( $\min =2,8.10^{6} ; \max =2,23.10^{7} \mathrm{UFC} / 100 \mathrm{~mL}$ ). Les échantillons de la deuxième campagne de prélèvements (P2) étaient les plus contaminés quelques soit le microorganisme considéré. Pendant toute la période on remarque une dominance des coliformes totaux. E. coli reste pour la plupart du temps en dessous des $10^{7}$ UFC/100mL. (Tableau 3)

Tableau 2: Évolution des microorganismes indicateurs de pollutions fécales dans la retenue $A$

\begin{tabular}{|c|c|c|c|c|}
\hline Retenue A (en UFC/100mL) & C. Totaux & C. Th & E. Coli & Strep. Fecaux \\
\hline P1 & $1,48 \cdot 10^{7}$ & $8,53.10^{6}$ & $4,73.10^{6}$ & $2,80.10^{6}$ \\
\hline P2 & $7,05.10^{7}$ & $5,17.10^{7}$ & $4,85.10^{7}$ & $2,23.10^{7}$ \\
\hline P3 & $1,96.10^{7}$ & $1,03.10^{7}$ & $6,90.10^{6}$ & $9,14.10^{6}$ \\
\hline P4 & $2,17.10^{7}$ & $1,36.10^{6}$ & $6,77.10^{5}$ & $4,55.10^{6}$ \\
\hline P5 & $4,47.10^{6}$ & $3,41.10^{6}$ & $3,32.10^{6}$ & $1,05.10^{6}$ \\
\hline P6 & $1,91.10^{7}$ & $1,66.10^{7}$ & $1,43.10^{7}$ & $1,13.10^{7}$ \\
\hline P7 & $7,16.10^{6}$ & $5,11.10^{6}$ & $1,88.10^{6}$ & $1,83.10^{6}$ \\
\hline P8 & $1,92.10^{7}$ & $4,55.10^{6}$ & $3,60.10^{6}$ & $7,21.10^{6}$ \\
\hline P9 & $1,10.10^{7}$ & $9,21.10^{6}$ & $8,21.10^{6}$ & $7,24.10^{6}$ \\
\hline $\mathrm{P} 10$ & $3,18.10^{7}$ & $1,90.10^{7}$ & $1,22.10^{7}$ & $1,04.10^{7}$ \\
\hline $\mathrm{P} 11$ & $1,07.10^{7}$ & $6,67.10^{6}$ & $5,63.10^{6}$ & $7,23.10^{6}$ \\
\hline $\mathrm{P} 12$ & $2,65.10^{7}$ & $1,16.10^{7}$ & $5,52.10^{6}$ & $9,46.10^{6}$ \\
\hline $\mathrm{P} 13$ & $1,85.10^{7}$ & $1,31.10^{7}$ & $9,27.10^{6}$ & $1,38.10^{7}$ \\
\hline P14 & $1,34.10^{7}$ & $1,23.10^{7}$ & $8,86.10^{6}$ & $3,00.10^{6}$ \\
\hline
\end{tabular}

$\mathrm{P}=$ Prélèvement ; C. Totaux = Coliformes Totaux ; C. Th= Coliformes Thermotolérants ; E. coli=Escherichia Coli ; Strep. Fécaux $=$ Streptocoques Fécaux 

Gourou au carrefour de l'Indénié à Abidjan en 2012.

Évolution des germes indicateurs de pollution fécale

de la retenue $B$ : Dans la retenue $B$, la concentration moyenne de Coliformes totaux était de $2,98.10^{7}$ UFC/100mL ( $\left.\min =6,43.10^{7} ; \max =1,16.10^{8} \mathrm{UFC} / 100 \mathrm{~mL}\right)$. Quant aux Coliformes thermololérants, $E$. coli et les streptocoques fécaux, les charges moyennes ont été respectivement de $2,19.10^{7} \mathrm{UFC} / 100 \mathrm{~mL}$ ( $\mathrm{min}=7,39.10^{6}$; $\max =1,10.10^{8} \quad$ UFC/100mL), $\quad 1,72.10^{7} \quad$ UFC/ $100 \mathrm{~mL}$ $\left(\min =1,9.10^{6} ; \max =1,00.10^{8} \mathrm{UFC} / 100 \mathrm{~mL}\right)$, et de $1,32.10^{7}$ UFC/100mL (min=7,8.105; $\left.\max =5,75.10^{7} \mathrm{UFC} / 100 \mathrm{~mL}\right)$. A l'inverse de $E$. coli et des coliformes qui baissent de $\mathrm{P} 1$ à P3, les concentrations des streptocoques fécaux augmentent. Pour la retenue $B$, les échantillons de la quatrième campagne de prélèvement (P4) étaient les plus contaminés. Pendant toute la période on remarque une dominance des coliformes totaux. (Tableau 4).

Tableau 3: Évolution des bactéries indicatrices de pollution fécales dans la retenue $B$

\begin{tabular}{lcccc}
\hline $\begin{array}{l}\text { Retenue B } \\
\text { en UFC/100mL }\end{array}$ & CT & C Th & E. coli & Strep. Fécaux \\
\hline P1 & $4,84.10^{7}$ & $1,25.10^{7}$ & $7,60.10^{6}$ & $7,8.10^{5}$ \\
P2 & $4,22.10^{7}$ & $3,85.10^{7}$ & $1,90.10^{7}$ & $1,14.10^{7}$ \\
P3 & $1,65.10^{7}$ & $1,00.10^{7}$ & $7,24.10^{6}$ & $1,60.10^{7}$ \\
P4 & $1,16.10^{8}$ & $1,1.10^{8}$ & $1,00.10^{8}$ & $5,75.10^{7}$ \\
P5 & $1,48.10^{7}$ & $1,33.10^{7}$ & $1,25.10^{7}$ & $6,50.10^{6}$ \\
P6 & $1,88.10^{7}$ & $9,94.10^{6}$ & $6,84.10^{6}$ & $7,01.10^{6}$ \\
P7 & $6,43.10^{6}$ & $9,24.10^{6}$ & $1,92.10^{6}$ & $2,73.10^{6}$ \\
P8 & $2,1.10^{7}$ & $1,56.10^{7}$ & $1,05.10^{7}$ & $1,77.10^{7}$ \\
P9 & $1,03.10^{7}$ & $9,24.10^{6}$ & $8,04.10^{7}$ & $1,40.10^{7}$ \\
P10 & $1,82.10^{7}$ & $1,13.10^{7}$ & $1,04.10^{7}$ & $8,20.10^{6}$ \\
P11 & $3,41.10^{7}$ & $1,93.10^{7}$ & $1,88.10^{7}$ & $1,83.10^{7}$ \\
P12 & $1,41.10^{7}$ & $7,4.10^{7}$ & $6,08.10^{7}$ & $7,75.10^{6}$ \\
P13 & $2,83.10^{7}$ & $2,23.10^{7}$ & $1,40.10^{7}$ & $1,48.10^{7}$ \\
P14 & $2,05.10^{7}$ & $1,83.10^{7}$ & $1,41.10^{7}$ & $2,30.10^{7}$ \\
\hline
\end{tabular}

$\mathrm{P}=$ Prélèvement $;$ C. Totaux $=$ Coliformes Totaux ; C. Th= Coliformes Thermotolérants ; E. coli=Escherichia Coli ; Strep. Fécaux= Streptocoques Fécaux

\section{DISCUSSION}

La présente étude a concerné 112 échantillons prélevés en 14 sorties. L'étude des paramètres bactériologiques a porté sur le dénombrement des germes indicateurs de pollution fécale. Tous les échantillons contenaient les germes tels les coliformes totaux, coliformes thermotolérants, les $E$. coli et les Streptocoques fécaux. La retenue $B$ est apparue plus pollué que la retenue $A$ même s'il faut noter que tous les deux ont des eaux de très mauvaise qualité avec des taux de bactéries supérieures au $10^{3} \mathrm{UFC} / 100 \mathrm{~mL}$ fixé par des directives de l'OMS (1989) pour le déversement des eaux usées sans risques dans l'environnement. Cette caractéristique des eaux usées avait été faite également par d'autres auteurs en Afrique (Adjahouinou et al 2014). En effet, les contaminations de ces retenues étaient superposables à celles obtenues dans les eaux usées urbaines tel que observé par Aboulouafa et al , (2002) et El Addouli et al ., (2008) au Maroc. Il en était de même des observations d'EI Ouali et al . (2014). Ces auteurs ont présenté des charges variant entre $10^{6} \mathrm{germes} / 100 \mathrm{~mL} 100 \mathrm{ml}$ et $2,6.10^{8}$
germes/100mL pour les coliformes totaux. Ceci est aussi vrai pour la Tunisie selon les travaux d'Eddabra (2011). Le niveau de contamination observé dans cette étude était supérieur aux taux obtenu par Adjahouinou et al (2014) au Bénin. Cette forte contamination pourrait s'expliquer par un grand déversement de déchets de toute sorte dans notre réseau de collecteurs. En effet, les collecteurs du bassin du gourou, initialement construit pour drainer les eaux fluviales, drainent à ce jour des ordures ménagères qui y sont jetées directement et les eaux usées issues de branchements anarchiques (Akossi, 2011). La saison pluvieuse au cours de laquelle ce travail a été mené, pourrait être aussi responsable de ces concentrations élevées selon Saad et al (2007), avec une augmentation des apports. Cependant la pluie aurait aussi un effet de dilution sur les charges microbiennes environnementales et pourrait avoir pour conséquence une sous-estimation des charges bactériennes [Saad et al , 2007]. Le rapport Coliforme Thermotolerant / Streptocoques fécaux (R) témoignant de l'origine des germes indicateurs de pollution 
fécale, était de 1,de1, 62 en moyenne pour la retenue $A$ et de 1,66 pour la retenue $B$. La pollution fécale était donc d'origine mixte dans les deux retenues selon Borrego et Romero (1982) cité par Larif et al , 2013. Cette origine pourrait s'expliquer par la nature diverse de la composition des eaux drainées par les collecteurs du bassin du gourou comme signifié par Akossi, 2011. En effet, les eaux usées des branchements anarchiques sont aussi bien d'origine domestique, hospitalière que des marchés et autres élevages installées aux abords des collecteurs. Cette origine incertaine de la contamination fécale des eaux usées est aussi mise en exergue par Larif (2013). Cependant, Aboulouafa et al (2002) a plutôt décrit une contamination d'origine humaine. Les eaux usées transitant par ces retenues sont rejetées dans la lagune Ebrié sans aucun traitement. Cette pollution microbienne pourrait être un risque aussi bien pour l'environnement que pour les riverains. En effet, ces charges bactériennes peuvent constituer un risque potentiel dans la mesure où ces retenues sont bordées d'habitations d'une part et

\section{CONCLUSION}

Les eaux des deux retenues étudiées présentent une mauvaise qualité bactériologique eue égard aux concentrations des germes indicateurs de pollution fécale. En effet les concentrations en $E$. coli, Streptocoques fécaux et coliformes totaux étaient largement supérieures aux normes OMS pour les eaux usées. II apparait alors plus que nécessaire de créer des filières de traitement de

\section{REMERCIEMENTS}

Les auteurs remerciement la direction de l'Institut Pasteur de Côte d'Ivoire, la Direction de l'Assainissement et du

\section{RÉFÉRENCES BIBLIOGRAPHIQUES}

Aboulouafa M, Hassan EH, Kharboua M, Berrichi A, 2002. Caractérisation physico chimique et bactériologique des eaux usées brutes de la ville d'Oujdou canal principal et Oued Bounaïm. Actes Inst.Agron.Vet. 22(3) : 143-150.

Adjahouinou DC., Yehouenou B, Liady MND, Fiogbe ED, 2014. Caractérisation bactériologique des eaux résiduaires brutes de la ville de Cotonou (Bénin). Journal of Applied Biosciences. 78: 6705-6713

Akossi Oreste Santoni. 2011. Optimisation des conditions d'évacuation des eaux pluviales du carrefour de l'indénié a la baie de Cocody. Master spécialisé génie sanitaire et environnement. Institut international d'ingénierie de l'eau et de l'environnement (2IE). 89 pages. d'autre part sont des lieux de récupération d'articles divers par les populations. Selon Vandermeersch (2006) [31], le coût global des maladies dues à la consommation et ingestion des produits provenant d'eaux polluées pourrait s'élever à plusieurs millions de dollars. La lagune Ebrié, utilisée par la population pour divers activités, notamment le maraichage (Wognin et al , 2013) pourrait être un lieu de propagation d'épidémie. La forte densité des $E$. coli, bactéries indicatrices les plus spécifiques d'une pollution fécale (CEAEQ, 2000 ; Edberg et al ., 2000), dans les eaux analysées, indique clairement leur contamination par des germes fécaux et par conséquent, le risque épidémiologique potentiel que représente leur rejet sans traitement (Servais et al ., 2006 ; Adjahouinou et al et al (2014)). En effet, la présence de ces entérobactéries, quoique non pathogènes dans leur grande majorité, doit faire sérieusement soupçonner celle de plusieurs autres microorganismes pathogènes tel que Salmonella $s p$ comme énoncé par Adjahouinou et al et al (2014).

ces eaux usées quand on sait qu'elles sont directement rejetées dans l'environnement. Ce qui pourrait réduire les risques de pollution environnementale d'une part et sanitaire d'autre part. Les résultats de ce travail posent aussi le problème du mode d'utilisation des collecteurs et des retenus d'eaux usées qui passent pour être de vrais dépotoirs.

Drainage, le Dr Adjé Christiane du Projet RETROCl et tous les Techniciens et employés des sites étudiés.

Anonyme, 2012. Tout sur les eaux usées :[ Disponible sur internet ]. http://www.sololiya.fr/index.php/tout_ sur_I_eau/eau_et_quotidien/activites_humaines/l es_menaces/2_les_eaux_usees consulté le 10 Juillet 2012

CEAEQ, 2000. Recherche et dénombrement des coliformes fécaux; méthode par filtration sur membrane. Centre d'Expertise en Analyse Environnementale, Gouvernement du Québec. 24 pp.

Cisse M, N'guessan F, Karamoko Y, Tigoli K, Dje Bi Dje F, Gourene G, 2011. Charge parasitaire des eaux usées du collecteur " gouro " traversant les communes d'Abobo, Adjamé et Cocody (district d'Abidjan). Les technologies de laboratoire. 6(25) : 96-106 
Côte d'Ivoire. Fonds Africain de développement (CI-FAD). 2010, Projet de gestion intégrée du bassin versant du Gourou Phase d'urgence, Résumé du projet, paragraphe 3, pp iii.

Edberg SC, Rice EW, Karlin RJ, Allen MJ, 2000. Escherichia coli: the best biological drinking water indicator for public health protection. Journal of Applied Microbiology. 88: 106S-116S.

Eddabra R, 2011. Évaluation de la contamination bactériologique des eaux usées des stations d'épuration du grand Agadir .Thèse, 146 $p$

El Addouil J, Chahlaoui A, Chafi A, Berrahou A, 2008. Suivi et analyse du risque lié à l'utilisation des eaux usées en agriculture dans la région de Meknes au Maroc. 2IE- Sud Sciences et Technologie. 16 : 29-35

El Ouali LA, Zanibou A, Bekhti K, Zerrouq F, Merzouki M, 2014. Contrôle de la qualité microbiologique des eaux usées domestiques et industrielles de la ville de Fès au Maroc. J. Mater. Environ. Sci. 5 (S1) : 2325-2332.

Fontaine 0,2008 . Nouvelles recommandations de l'OMS pour la prise en charge clinique de la diarrhée. Paediatrica 19 (5) : 25-27

Kotloff KL, Blackwelder WC, Nasrin D, Nataro JP, Farag $\mathrm{TH}$, van Eijk A, Adegbola RA, Alonso PL, Breiman RF, Faruque AS, Saha D, Sow SO, Sur D, Zaidi AK, Biswas K, Panchalingam S, Clemens JD, Cohen D, Glass RI, Mintz ED, Sommerfelt H, Levine MM, 2012. The Global Enteric Multicenter Study (GEMS) of diarrheal disease in infants and young children in developing countries: Epidemiologic and clinical methods of the case/control study. Clin. Infect. Dis. 55: S232S245

Larif M, Soulaymani A, Hnach M, El Midaoui A. 2013. Contamination spatio-temporelle d'origine hydrique de l'oued Boufekrane dans la région de Meknès-Tafilalt (Maroc). Int. J. Biol. Chem. Sci. $7(1): 172-184$

Levantesi C, La Mantia R, Masciopinto C, Böckelmann U, Ayuso-Gabella MN, Salgot M, Tandoi V, Van
Houtte E, Wintgens T, Grohmann E. 2010. Quantification of pathogenic microorganisms and microbial indicators in three wastewater reclamation and managed aquifer recharge facilities in Europe. Sci. Total Environ. 408: 49234930.

Ouattara PJ-M, 2005. Fonctionnement des grands collecteurs d'eaux pluviales dans cinq communes d'Abidjan-Nord (Abobo-Adjamé-AttécoubéCocody) et auto-épuration des eaux dans le collecteur Gouro. Mémoire de DEA, Université Abobo-Adjamé, Abidjan, Côte d'Ivoire, 65p

Saad HB, Nassif N, Antoine G, Samrani E, Daoud R, Medawar S, Ouaïni N, 2007. Suivi de la qualité bactériologique des eaux de surface. Revue des sciences de l'eau / Journal of Water Science, 20 (4) : 341-352.

Servais P, Garcia-Armisen T, Lizin P, Mercier P, Anzil A, 2006. Modélisation de la dynamique des indicateurs de qualité microbiologique en estuaire de Seine. Rapport scientifique Seine-Aval 3. $11 \mathrm{pp}$.

Servais P., Billen G., Tamara G.-A., George I., Goncalvez A. et Thibert S. 2009. La contamination microbienne des eaux du bassin de la Seine. Eau Seine Normandie, Programme PIREN-SEINE.

Vandermeersch S, 2006 Étude comparative de l'efficacité des traitements d'épuration des eaux usées pour l'élimination des micro-organismes pathogènes. Université libre de Bruxelles ; Mémoire de DES. $54 \mathrm{P}$.

Wayou TP, 2010. Diagnostic du fonctionnement du réseau d'assainissement de la commune de Yopougon : cas du quartier Niangon en Côte d'Ivoire. Master I de sciences et gestion de l'environnement. Université d'Abobo- Adjamé Côte d'Ivoire.

Wognin AS, Ouffoué KS, Assemand EF, Tano K, KoffiNevry R, 2013. Perception des risques sanitaires dans le maraîchage à Abidjan, Côte d'Ivoire. Int. J. Biol. Chem. Sci. 7(5): 1829-1837. 